\title{
METHOD FOR DETERMINING MOTION TRAJECTORIES OF CHARACTERISTIC POINTS REGISTERED BY A VIDEO CAMERA
}

\author{
T. Piatkowski*, M. Wolski ${ }^{* *}$, P. Osowski ${ }^{* * *}$
}

\begin{abstract}
The paper presents a method of simultaneous tracking of motion trajectories of many characteristic points based on the analysis of images recorded with a high-speed digital camera. In this method we applied functions from the Video and Image Processing Blockset toolbox in the Matlab environment (for distinguishing characteristic objects from the background of the image) and own function designed for assigning recognized points to the appropriate trajectories. The effectiveness of the method was confirmed during experimental tests of tracing four characteristic points in the process of unit loads sorting with the flexible fence of rotary motion.
\end{abstract}

Keywords: image processing, BLOB, sorting process, active fence, package

\section{Introduction}

The computer image analysis technique finds many practical applications today, e.g. in the detection of surface defects in industrial quality control of products (Chuanxia, 2017), cell counting in biological preparations observed under a microscope (Alreza, 2016), the areas classification in remote sensing (Galda, 2003).

The basic feature of computer image analysis is the non-invasive (non-contact) nature of the examination of objects and physical phenomena. This feature causes that during tests, we neither interfere in the properties of the object nor its functioning. In case of a high speed camera use (Osowski, 2017), the method is also suitable for registering and analyzing fast-changing processes, e.g. the process of automatic sorting of unit loads (Piatkowski, 2009, 2014) or crack initiation (Tomaszewski, 2012).

Nowadays there are computer aided engineering programs (e.g. Matlab, Scilab - Campbell, 2006 and Gonzales, 2004), which contain toolboxes with functions to perform basic image analysis. However, they do not have algorithms to simultaneously track the motion paths of several selected characteristic points. The need to develop such algorithm occurs in case of experimental tests of kinematic and dynamic parameters of multibody mechanical systems containing flexible elements (Wolski, 2017).

The main aim of the paper is to propose an algorithm for simultaneous tracking of motion paths of many characteristic points. The experimental verification of the developed algorithm will be carried out on the example of the recording analysis of the sorting process of the packaging with the flexible active fence (Fig. 1) registered by means of the high-speed camera AOS Q-Pri.

Assoc. Prof. Tomasz Piatkowski, PhD.: Faculty of Mechanicla Engineering, UTP University of Technology and Science, Street Kaliskiego 7; 85-796, Bydgoszcz; PL, tomasz.piatkowski@utp.edu.pl

** MSc Miroslaw Wolski: Faculty of Mechanicla Engineering, UTP University of Technology and Science, Street Kaliskiego 7; 85-796, Bydgoszcz; PL, miroslaw.wolski@utp.edu.pl

*** MSc Przemyslaw Osowski: Faculty of Mechanicla Engineering, UTP University of Technology and Science, Street Kaliskiego 7; 85-796, Bydgoszcz; PL, przemyslaw.osowski@utp.edu.pl 


\section{Registration method for motion trajectories of characteristic points}

The algorithm (Fig. 2) for the simultaneous tracking of the motion trajectories of several characteristic points was developed to evaluate the course of the sorting process of packaging with the flexible active fence which is often applied in logistic centers (Fig. 1). In this assessment, it is important to determine the actual trajectories, velocities and accelerations of the motion of the fence and packaging from the point of view of efficiency, reliability and safety of the scrapped packages. The characteristic points are marked in red, assuming that this color will be as little as possible registered in the background.

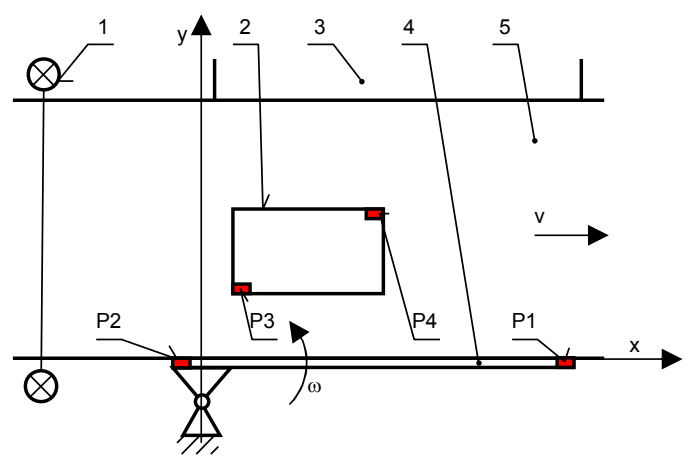

Fig. 1: Laboratory stand for automatic sorting of packages: P1, P2, P3, P4-characteristic points, 1 -light barrier, 2 - packaging, 3 -skid, 4 -flexible fence, 5 - main conveyor, $\omega$-angular velocity of the fence, $v$ - linear velocity of the conveyor.

In Fig. 2 is shown an algorithm for image processing developed in the Matlab Simulink environment. The main part of this algorithm consists of six blocks: five $(1-4,6)$ are taken from the toolbox "Video and Image Processing Blockset" and one (5) represents authors' own elaboration. The block (5) is responsible for the assignment of points to the relevant motion trajectories (Fig. 3). The assigning process is based on the calculation of the distances between the points of the current and previous frames - according to the comments shown in Fig. 3. In order to achieve better color selectivity, three-channel RGB images are converted to $\mathrm{YCbCr}$ format.

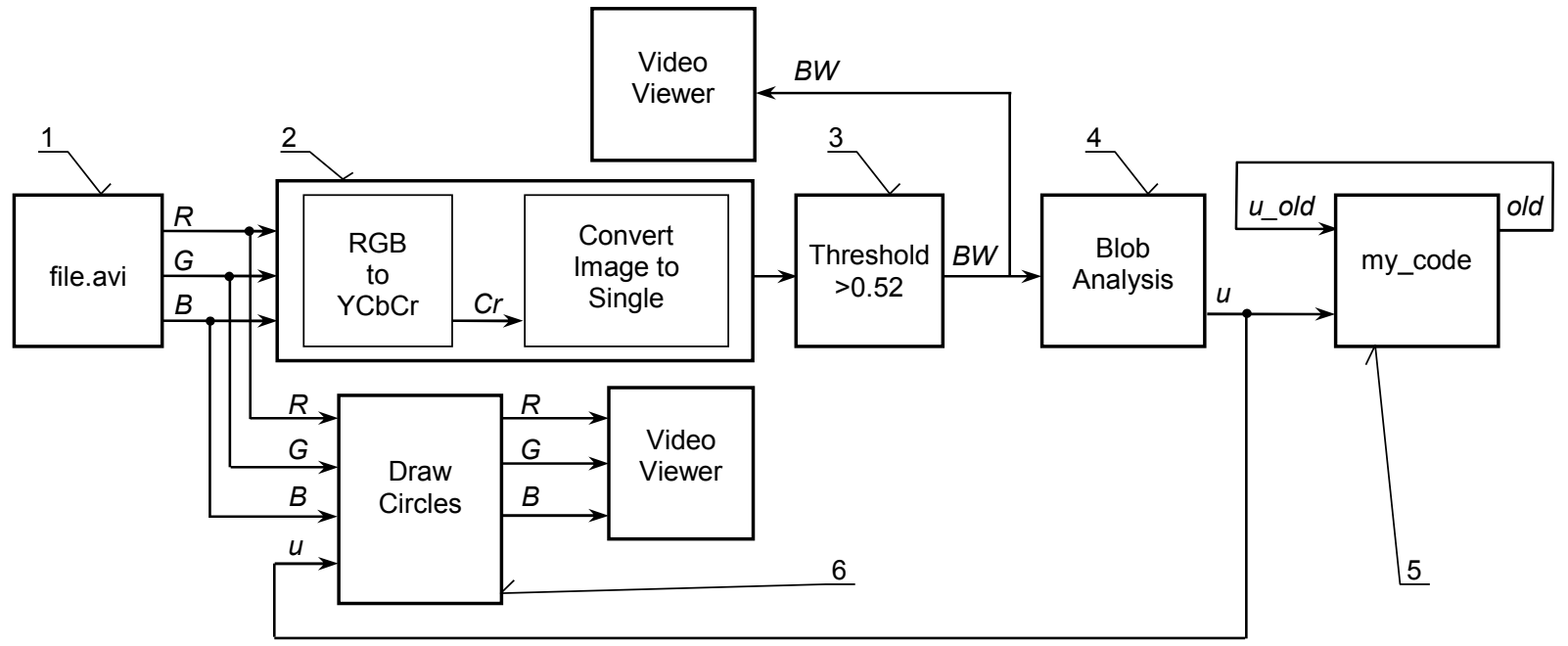

Fig. 2: Algorithm for the motion trajectories identication of the characteristic points: 1 -video file reading, 2 - image conversion from $R G B$ format to $\mathrm{YCbCr}$ with $\mathrm{Cr}$ channel selection, 3 - the $\mathrm{Cr}$ channel conversion to black and white (logical) image based on Threshold value, 4-search for characteristic points, 5 - assigning points to the appropriate motion paths, 6 -combining the original image with the circles identifying the detected points. 


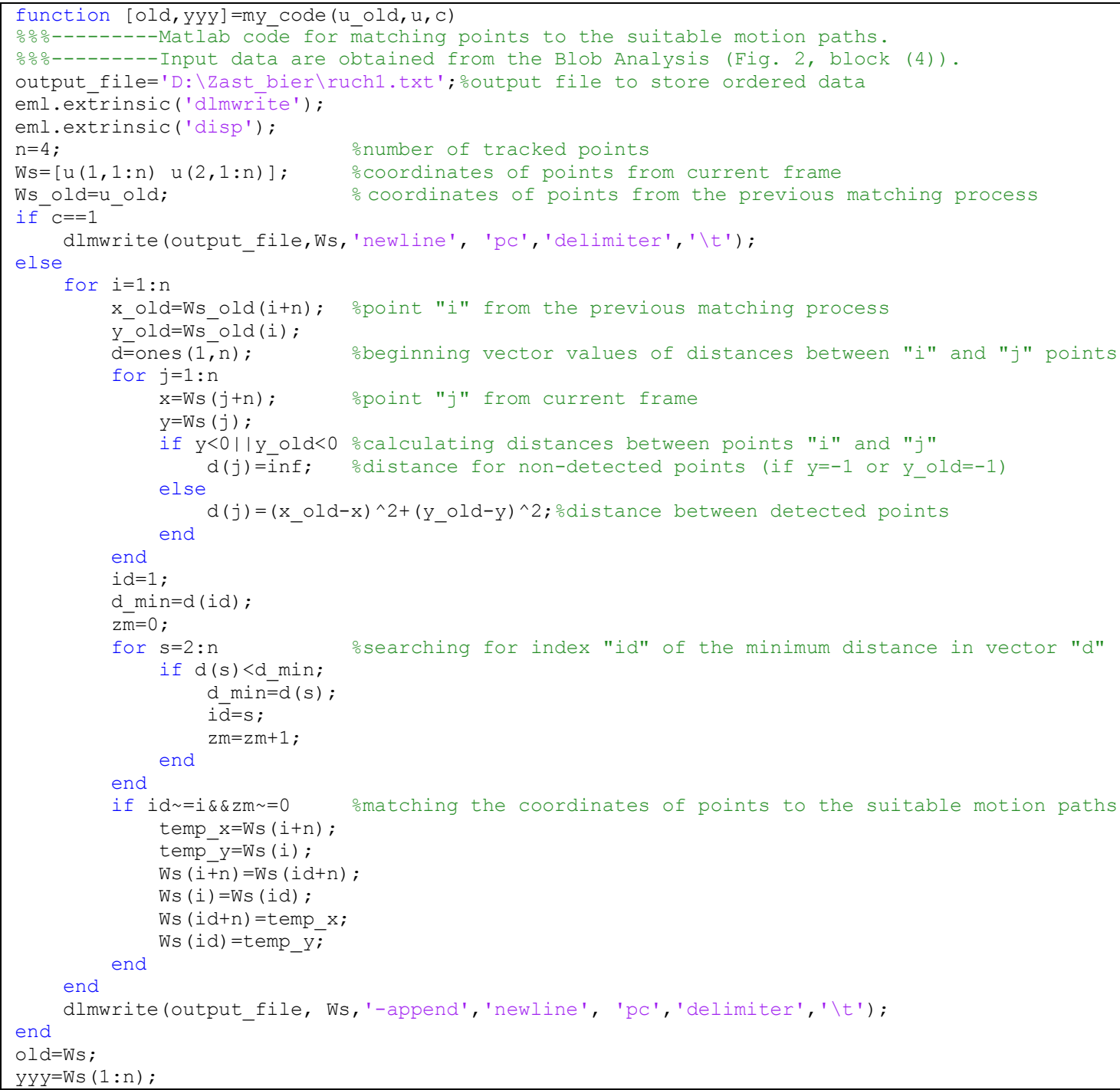

Fig. 3: Code of my_code function - a block (5) in Fig. 2.

\section{The results of experimental tests}

Fig. 4a shows the original image recorded by a digital camera with attached text and circles identifying detected points. Fig. $4 \mathrm{~b}$ presents a logical image obtained after image conversion of single-channel $\mathrm{Cr}$ and application of the threshold value Threshold $>0.52$ (selected by trial and error method). The white color (logical truth) represents the recognized characteristic points.
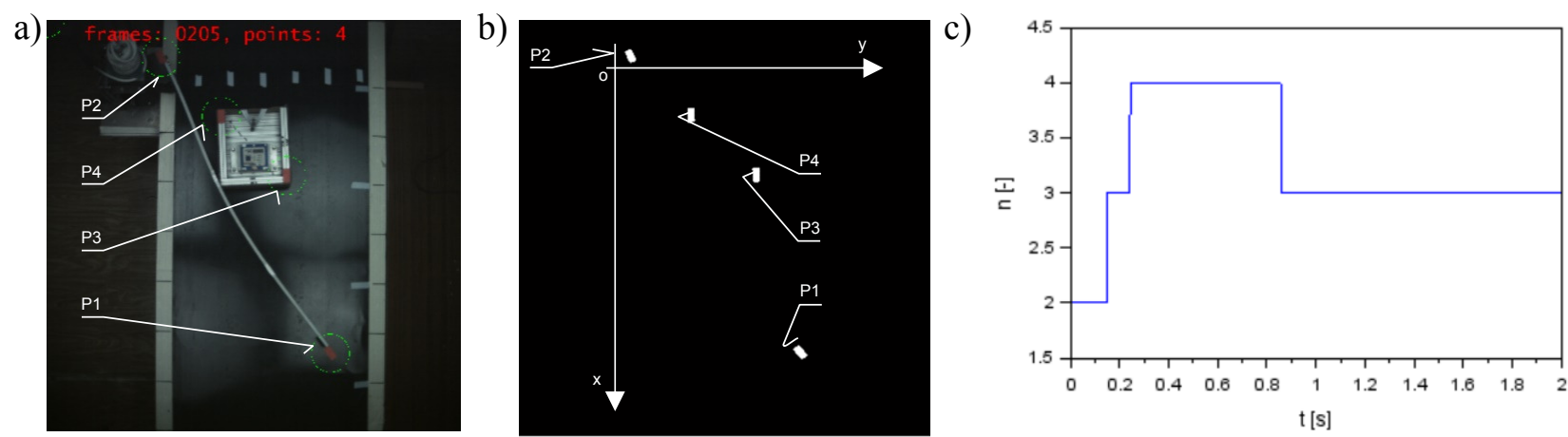

Fig. 4: Analysis results of the video recording - part 1: a) the image with the marked circles identifying detected points, b) black and white image with selected points $\left.P_{1} \div P_{4}, c\right)$ the number of recognized characteristic points in the function of time. 
a)

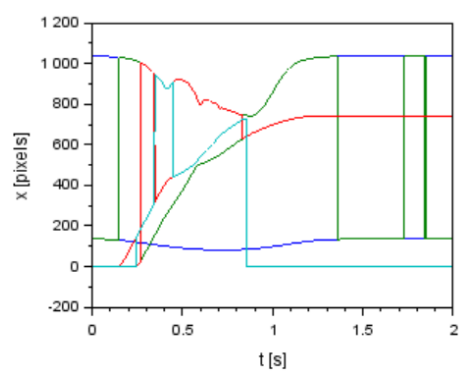

b)

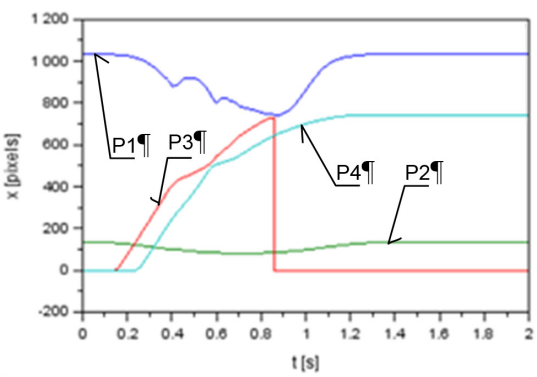

c)

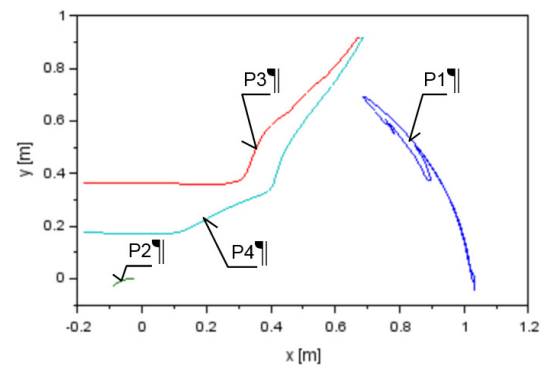

Fig. 5: Analysis results of the video recording - part 2: a) coordinates $x(t)$ of motion paths $P_{1} \div P_{4}$ detected by the block (4) from Fig. 2, b) ordered coordinates $x(t)$ of motion paths of points according to block (5) in Fig. 2, c) motion paths of points in the oxy coordinate system.

It results from the analysis of Fig. 4c that the number of recognizable characteristic points changes during the image processing. This variability is caused by appearance and disappearance of the packaging in the camera "field of view". Fig. 5a shows raw data obtained directly from the block (4), that after being processed by block (5) are presented in the Fig. 5b, c. These plots (Fig. 5b, c) demonstrate that the proposed code (Fig. 3), correctly assigns characteristic points $P_{1} \div P_{4}$ to the appropriate paths, regardless of the number of visible points.

\section{Conclusions}

Based on the experimental verification, it can be concluded that the proposed method of the motion paths determination of many characteristic points (theoretically infinitively many) with the use of digital image processing toolboxes accessible in popular mathematical software, combined with own procedures is effective. This method also correctly allows assigning the motion paths to characteristic points, which are not always visible in the frames. The motion paths are basis for determining any derivative quantities (e.g.: relative or absolute positions, velocities and accelerations), allowing for in-depth analysis and evaluation of the influence of adopted control parameters on utility process realized by the mechanical device.

\section{References}

Alreza, Z. and Karimian, A. (2016) Design a new algorithm to count white blood cells for classification Leukemic Blood Image using machine vision system. 6th International Conference on Computer and Knowledge Engineering (ICCKE), Mashhad, Iran, 251-256.

Campbell, S.L., Chancelier, J.P. and Nikoukhah, R. (2006) Modeling and Simulation in Scilab/Scicos, Springer, New York.

Chuanxia, J. and Yinhui A. (2017) Automatic surface defect detection for mobile phone screen glass based on machine vision. Applied Soft Computing, 52, 348-358.

Galda, H. (2003) Development of a segmentation method for dermoscopic images based on color clustering, Kobe University.

Gonzales R. C., Woods, R.E. and Eddins, S.L. (2004) Digital Image Processing Using MATLAB. Pearson Prentice Hall, Upper Saddle River, New Jersey.

Osowski, P., Wolski, M. and Piatkowski, T. (2017) Verification of velocity measurement methods by high speed camera and accelerometer on example of impact tester, In: Engineering Mechanics 2017, Brno University of Technology, Brno, 742-745.

Piatkowski, T. (2009) Model and analysis of the process of unit-load stream sorting by manipulator with torsional disks. Journal of Theoretical and Applied Mechanics, 4(47), pp. 871-896.

Piatkowski, T., Sempruch, J. and Tomaszewski, T. (2014) Dynamics of a sorting process with a stream of discrete impact loads, Transactions of the Canadian Society for Mechanical Engineering, 1(38), 139-154.

Shapiro, L. G., Stockman, G.C. (2001) Computer Vision. Prentice-Hall Inc. Upper Saddle River, New Jersey.

Tomaszewski T., Sempruch, J. (2012) Determination of the fatigue properties of aluminum alloy using mini specimen. Materials Science Forum, 726, pp. 63-68.

Wolski, M., Piątkowski, T. and Osowski, P. (2017) Rotary motion selected control methods analysis for paddle sorters arms, In: Engineering Mechanics 2017, Brno University of Technology, Brno, 1062-1065. 